\title{
3 Research Square

\section{Maxitrol Ophthalmic Ointment Versus Prednisolone Acetate and Gentamicin Eye Drops after Pars Plana Vitrectomy: A Retrospective Chart Review}

Shambhawi Thakur, MS ( $\nabla$ shami.thakur96@gmail.com )

Eastern Virginia Medical School

Alan L. Wagner, MD, FACS, FICS

Wagner and Kapoor Research Institute

Shawna Gallagher

Wagner and Kapoor Research Institute

Kapil G. Kapoor, MD, FACS, FICS

Wagner and Kapoor Research Institute

\section{Research Article}

Keywords: gentamicin, infection, maxitrol, postoperative inflammation, PPV, prednisolone acetate, vitrectomy

Posted Date: February 18th, 2022

DOI: https://doi.org/10.21203/rs.3.rs-1363736/v1

License: () (1) This work is licensed under a Creative Commons Attribution 4.0 International License. Read Full License 


\section{Abstract}

\section{Background}

Maxitrol ophthalmic ointment is a combination anti-inflammatory and antibiotic agent commonly used to manage post-operative inflammation and infection. In July 2019, Altaire Pharmaceuticals, Inc. announced the recall of 99 eye-care products as a precautionary measure due to quality assurance concerns. Recalled products included the generic Maxitrol ophthalmic ointment which is covered by most insurance plans, including Medicare and costs around $\$ 20$ in the United States. The recall led to its shortage, and consequently, patients who were prescribed the Maxitrol ophthalmic ointment for their post-operative regimen were obliged to purchase the branded version at its full out-of-pocket cost (\$213) as it is not covered by many insurance plans, including Medicare.

The Prednisolone Acetate and Gentamicin eye drops have a combined retail price of $\$ 56$, but are covered by Medicare and most insurance plans. They have been shown to be safe and effective against postsurgical inflammation and infection after cataract surgeries. In this study, we aim to compare the safety and efficacy of Maxitrol ophthalmic ointment and combination Prednisolone Acetate/Gentamicin eye drops on post-operative ocular inflammation and prevention of infection after pars plana vitrectomy (PPV).

\section{Main Body}

This was a retrospective study including all patients that underwent PPV for macular hole repair, epiretinal membrane, vitreous debris removal, vitreous hemorrhage, and severe floaters from February 2019 to 2021. Patients with retinal detachment surgeries and diabetes were excluded due to wider variation in inflammation. The medical records of all surgical patients were thoroughly reviewed for the six-month postoperative period and clinical data points regarding infection and inflammation were recorded. Fischer's exact test was utilized to analyze the data.

Of 337 PPV surgeries, 178 eyes fulfilled the inclusion criteria. During the six-month post-operative period, there was no statistical difference in post-operative rates of infection or inflammation $(p=1.0)$.

\section{Conclusion}

Both treatments were equally as safe and effective after PPV. This data is critical in allowing clinicians to optimize post-operative regimens, allowing flexibility regarding back orders of medication, sensitivity to ease of application, and awareness of cost-effectiveness.

\section{Background}

Vitrectomies have a low risk of complications, including infection, retinal detachment, increase or decrease in intraocular pressure (IOP), cataract formation, loss of vision, macular edema and intraocular inflammation. ${ }^{1}$ Excellent post-operative care remains a cornerstone of minimizing post-operative 
complications following PPV. While various regimens for post-operative care have evolved, universally at least a steroid and antibiotic are utilized.

Maxitrol (Neomycin $3.5 \mathrm{mg}$, Polymyxin B Sulfates 10,000 IU/g and Dexamethasone $0.1 \% \mathrm{w} / \mathrm{w}$ Ophthalmic Ointment) is a combination anti-inflammatory and antibiotic agent commonly used to manage postoperative inflammation and infection. ${ }^{2}$ The combination ointment is reported to have greater patient compliance and convenience due to its correct volume of drug delivery and retention, and compatibility of drugs when both types of drugs are combined into one formulation. The brand name version of the drug is manufactured by Novartis Pharmaceuticals Canada Incorporated. It costs around \$213 in the United States and is not covered by many insurance plans, including Medicare. ${ }^{3}$ The generic version of Maxitrol ophthalmic ointment, known as Neo-Poly Dex, is manufactured by Perrigo Company (shipped exclusively from Altaire Pharmaceuticals Incorportated) and Bausch Health. ${ }^{4}$ The $3.5 \mathrm{~g}$ tube contains the same dosage of active ingredients: Neomycin $3.5 \mathrm{mg}$, Polymyxin B Sulfates 10,000 International Unit/g and Dexamethasone $0.1 \%$ weight to weight. ${ }^{5}$ It is covered by most insurance plans, including Medicare and costs around $\$ 18$ in the United States. ${ }^{3}$

In July 2019, Altaire Pharmaceuticals, Incorporated announced the recall of 99 eye-care products as a precautionary measure due to quality assurance concerns. ${ }^{6}$ Recalled products included Neo-Poly Dex ointment which resulted in a shortage of the generic Maxitrol product. Consequently, patients who were prescribed the Maxitrol ophthalmic ointment for their post-operative regimen were obliged to purchase the branded version at its full out-of-pocket cost.

Unfortunately, Altaire Pharmaceuticals Incorporated did not have a timeline for the resupply of Neo-Poly Dex ointment. Since drug shortages cause drug prices to increase twice as quickly, the situation put a high cost burden on patients. ${ }^{7}$ It also put ophthalmologists in a difficult position as higher prescription costs lead to medication non-adherence, and a subsequent increase in the risk of infections. In February 2020, the providers at Wagner Macula \& Retina Center (WMRC) made the decision to temporarily substitute Maxitrol ophthalmic ointment with Prednisolone Acetate and Gentamicin eye drops to ensure better postoperative outcomes for their patients. The two bottles of eye drops have a combined retail price of $\$ 56$, but are covered by Medicare and most insurance plans. ${ }^{8,9}$ Moreover, they have been shown to be safe and effective against post-surgical inflammation and infection after cataract surgeries. ${ }^{10}$

In this study, we aim to compare the efficacy of the two post-operative prophylactic regimens as no studies have investigated this specific topic.

\section{Methods}

\section{Study Design}

This study was a two-year retrospective chart review for PPV surgeries that occurred from February 2019 to February 2021. The main objective of the study was to compare the efficacy and safety of Maxitrol 
(ophthalmic ointment) and the concomitant use of Prednisolone Acetate and Gentamicin (eye drops) in the treatment of post-operative intraocular inflammation and prevention of infections following PPV.

The providers at WMRC started prescribing Prednisolone Acetate and Gentamicin eye drops from February 11,2020 , and therefore, the data was gathered from both treatment groups for one year each. The prescription for both regimens were to instill the medication 4 times a day for the first week, 3 times a day for the second week, twice a day for the third week, and once a day for the fourth week.

A chart review of 337 surgeries of patients ranging between 40 and 92 years old was conducted. AthenaNet, the cloud-based Electronic Health Record application was used to build a comma-separated values report of patients who underwent vitreoretinal surgeries with the procedure codes 67036 (vitrectomy, mechanical, pars plana approach), 67039 (vitrectomy, mechanical, pars plana approach; with focal endolaser photocoagulation), and 67042 (vitrectomy membranectomy macular hole) that were performed by Kapil G. Kapoor, MD, FACS, FICS from February of 2019 to February 2021. Charts were excluded from the study if they had: 1) concomitant systemic and ocular pathologies (diabetes and uveitis); 2) pars plana vitrectomy for retinal detachment, endophthalmitis, and vitreous prolapse; 3 ) post-cataract removal surgeries (repositioning of IOL, subluxation of the lens, anterior dislocation of the lens, removal of dislocated IOL, and removal of retained lens material or fragments); and 4) use of intra-operative intravitreal injection.

The data was collected from NexTech IntelleChartPRO and was recorded in password-protected Microsoft Excel. The variables included patient ID, patient name, patient's date of birth, procedure code, date of surgery, type of surgery, medication prescribed, and notes on inflammation, infection rates or exclusion criteria, where applicable.

All chart notes were thoroughly reviewed for up to six months of the post-surgical period. The patients who undergo surgery at WMRC are scheduled for appointments on post-operative day 1, 7, 30, 90, and 180 . More appointments were scheduled to monitor the patients, if necessary, on a case-by-case basis. Evidence of post-operative infection and intraocular inflammation served as the primary efficacy parameters of the treatment groups. The notes under the General section of the Anterior Segment of the operative eye on the NexTech IntelleChartPRO were evaluated for the presence or absence of post-operative infection. Postoperative intraocular inflammation was analyzed through the chart notes in the Impression/Plan section and was considered 'acute' if it occurred within the first six weeks after the surgery and was considered as 'delayed rebound inflammation' if it occurred after six weeks and before six months after the surgical procedure. Anterior Chamber inflammation was also investigated through the notes under Anterior Chamber on NexTech IntelleChartPRO where the flare and cells were recorded. The anterior chamber inflammation was rated from 0 to 4 based on the Standardization of Uveitis Nomenclature (SUN) grading system. Elevation of IOP was monitored to assess the safety parameters of the medications.

\section{Statistical Analysis}

Statistical tests of the study were determined to be descriptive because exploratory data analysis was used to investigate the data. The categorical variables of the study were analyzed through counts and 
percentages of the included charts. Where applicable, Fischer's exact test was selected to compare the categorical data at a 5\% significance level. Statistical analysis was performed in Microsoft Excel Version 16.16.3 using Data Analysis.

\section{Results}

A total of 178 charts were included in the chart review: 88 subjects were prescribed Maxitrol ophthalmic ointment and 90 subjects were prescribed Prednisolone Acetate and Gentamicin eye drops.

None of the patients from either treatment groups developed an infection in the six-month post-operative period. Two patients from each treatment group had post-surgical intraocular inflammation. Thus, $2.27 \%$ of the Maxitrol ophthalmic ointment group and $2.22 \%$ of the Prednisolone Acetate and Gentamicin eye drops group developed inflammation within six months of PPV. All four patients were determined to have 'delayed rebound inflammation' as the inflammation occurred between the six weeks and six months of the post-surgical period. Accordingly, $97.73 \%$ of the Maxitrol ophthalmic ointment group and $97.78 \%$ of the eye drops treatment group did not show signs of inflammation. The Fischer's exact test yielded a p-value of 1.000 which shows that there was no statistically significant difference between the two groups.

Anterior chamber inflammation was also detected in a few patients. In the Maxitrol ophthalmic ointment treatment group, a total of $1.5+$ cell and $1+$ flare was observed in the anterior chamber among two patients. In the Prednisolone Acetate and Gentamicin eye drops treatment group, $2+$ flare was observed in the anterior chamber of one patient. Anterior chamber inflammation was not observed in any of the other patients $(S U N$ grade $=0)$ showing that it was comparable between the two treatment groups.

Regarding the safety parameters to evaluate the safety of the treatments, no post-surgical adverse complications were noted. Elevation of IOP (>22 mmHg) was recorded for only two patients in the eye drops treatment group which improved within two weeks after the addition of Cosopt or Timolol eye drops to the treatment regimen.

\section{Discussion}

PPV surgery has played a major role in the advancement of treating conditions of the posterior segment of the eye, and monitoring post-operative patients for long-term complications is important to prevent serious complications, such as, intraocular inflammation and eye infection. ${ }^{1}$ Maxitrol ophthalmic ointment has long been a preferred treatment post-operatively as it combines anti-inflammatory and antibiotic properties in one formulation. ${ }^{2}$ While there was an underlying presumption that ointment had better absorption and thus, would result in a more optimal regimen with further reduction in inflammation/infection risk, the present data suggests otherwise. The data clearly shows no measurable difference in these rates which now widens the clinician's flexibility post-operatively.

Maxitrol's main disadvantage is its cost, as the branded version of the ointment costs roughly ten times more than the generic version. In addition, most insurance plans and Medicare do not cover the branded 
version of Maxitrol. Although most pharmacies carry the generic version of the Maxitrol ophthalmic ointment, the medication shortage in 2019-2020 caused the prices to be too expensive for the patients, since most patients getting vitrectomy in the United States tend to be on Medicare. Since drug shortages are unpredictable and high prescription costs can lead to medication non-adherence, it is important to have reliable treatment alternatives that are affordable and equally as effective.

In this study, the post-operative intraocular inflammation (including anterior chamber inflammation) between the two groups was almost identical. Additionally, no post-surgical infection was noted in either of the two groups. Our results showed no statistical differences between the ointment and the eye drops. Also, no significant adverse events or safety concerns occurred in any of the patients which indicates that the treatments are well tolerated. The findings are consistent with the study that suggested Prednisolone Acetate-Gentamicin eye drops have a positive therapeutic effect on post-operative patients of cataract surgeries. ${ }^{10}$

To illustrate this more granularly, one of the patients who underwent a PPV for macular hole for her right eye on September 13, 2016 was prescribed the Maxitrol ophthalmic ointment for her post-operative treatment. Almost five years later, a macular hole arose in her left eye which was operated on January 12, 2021 via PPV by the same physician. For the 2021 PPV, however, she was prescribed the Prednisolone Acetate and Gentamicin eye drops. After reviewing her charts from the six-month post-operative periods of both the surgeries, it was clear that both the prescriptions had effective outcomes as she did not develop an infection or post-surgical inflammation after either of the surgeries.

A main limitation of the study was that the number of events (inflammation and infection rates) was lower than expected which decreased the power of the study. However, due to the descriptive nature of the study, the sample size was considered to be enough. Additionally, the exclusion of certain ocular and systemic diagnoses might have introduced selection bias. However, the inclusion of diabetic patients, retinal detachment surgeries, and the other conditions listed in the exclusion criteria would be confounding factors in the study. These confounders can skew the data as they carry a higher risk of inflammation and infection.

\section{Conclusions}

In conclusion, both treatments were equally as safe and effective in the treatment of ocular inflammation (including anterior chamber inflammation) and prevention of bacterial infection after PPV. This data is critical in allowing clinicians to optimize a post-operative treatment regimen for each patient after PPV, allowing flexibility regarding medication shortages, sensitivity to ease of application, and awareness of cost-effectiveness, given their clinical interchangeability. Additional randomized controlled trials will help further clarify the optimal post-operative regimen following PPV.

\section{List Of Abbreviations}

PPV: Pars Plana Vitrectomy 
IOP: Intraocular Pressure

WMRC: Wagner Macula \& Retina Center

SUN: Standardization of Uveitis Nomenclature

\section{Declarations}

Ethics approval and consent to participate: IRB approved by Eastern Virginia Medical School

Consent for publication: Not applicable

Availability of data and materials: The data that support the findings of this study are available from Wagner Macula \& Retina Center, but restrictions apply to the availability of these data, which were used under license for the current study, and so are not publicly available.

The authors declare that they have no competing interests or funding resources.

Author's contributions: ST analyzed and interpreted the data, and was a major contributor in writing the manuscript. ALW and KGK performed the patient eye examinations and helped with the design of the study. SG was a major contributor of the study design. All authors read and approved the final manuscript.

Acknowledgments: Not applicable

\section{References}

1. Vitrectomy - The American Society of Retina Specialists. Accessed June 21, 2021. https://www.asrs.org/patients/retinal-diseases/25/vitrectomy

2. Maxitrol Ointment. Accessed June 28, 2021. https://dailymed.nlm.nih.gov/dailymed/fda/fdaDrugXsl.cfm?setid=22c6d2db-85af-4a3b-a01480d901a86717\&type=display

3. Maxitrol Prices, Coupons \& Savings Tips. GoodRx. Accessed August 23, 2021. https://www.goodrx.com/maxitrol

4. Generic Maxitrol Availability. Drugs.com. Accessed June 24, 2021. https://www.drugs.com/availability/generic-maxitrol.html

5. Neomycin-and-Polymyxin-B-Sulfates-and-Dexamethasone-Oint-A-9114102-9114002.pdf. Accessed June 28, 2021.

https://www.bausch.com/Portals/77/-/m/BL/United\%20States/Files/Package\%20Inserts/Pharma/RxGenerics/Neomycin-and-Polymyxin-B-Sulfates-and-Dexamethasone-Oint-A-9114102-9114002.pdf

6. UPDATE: Massive Altaire recall sweeps 99 eyecare products off shelves. American Academy of Ophthalmology. Published July 8, 2019. Accessed June 28, 2021. https://www.aao.org/headline/alertmassive-altaire-recall 
7. Drug shortages put ophthalmologists and their patients in difficult positions. Accessed June 28, 2021. https://www.healio.com/news/ophthalmology/20190814/drug-shortages-put-ophthalmologists-andtheir-patients-in-difficult-positions

8. Prednisolone Acetate Prices and Prednisolone Acetate Coupons - GoodRx. Accessed June 28, 2021. https://www.goodrx.com/prednisolone-acetate

9. Gentamicin Prices and Gentamicin Coupons - GoodRx. Accessed June 28, 2021. https://www.goodrx.com/gentamicin

10. Carriker F, Liebowitz S, Nees $\mathrm{O}$, et al. Prednisolone acetate-gentamicin combination following cataract surgery. Ann Ophthalmol. 1987;19(7):247-250. 